\title{
SIMPLE PARAMETRIZATION SCHEME FOR ELECTRIC DIPOLE INTENSITIES
}

\author{
Z. GAJEK \\ W. Trzebiatowski Institute of Low Temperature and Structure Research \\ Polish Academy of Sciences \\ P.O. Box 937, 50-950 Wrocław 2, Poland
}

\begin{abstract}
A simple phenomenological model for the electric dipole intensities of $n f \rightarrow n f$ transitions is proposed. The model is derived by adopting of the common angular overlap model to the generalized Judd-Ofelt theory of the static coupling mechanism. It leads to reduction of a number of the effective intensity parameters to six, three of which correspond to the $f \rightarrow d$ excitations and the remaining to the $f \rightarrow g$ ones. Calculations performed for some lanthanide complexes show that the model, despite its inborn crudeness, provides satisfactory results and may be a convenient tool for a preliminary estimation of intensities. Regularity of the obtained parameters indicates a possibility of further reduction of their number.
\end{abstract}

PACS numbers: 78.20.Bh, 78.20.Dj

\section{Introduction}

Large number of free parameters and limited understanding of their physical origin makes difficult an application of the general, mechanism independent, parametrization schemes for $n f \rightarrow n f$ electric dipole intensities [1-3]. All problems with both phenomenological and $a b$ initio determination of electronic structure of metal ion in crystal remain but this time at the level of at least the second order perturbation theory. Therefore, it is not surprising that the calculations of the intensity parameters from the first principles reported so far are not overthorough though they convey an idea of relative importance of various mechanisms of both dynamic and static origin $[4,5]$. Recent theoretical studies of the two-photon process evidenced an important role of third order terms [6]. It seems that more reliable treatment in a function basis not truncated to metal orbitals goes beyond present computational power.

An alternative method consists of an adoption of simplified models based on the superposition approximation [1]. This approximation reduces the number of independent intensity parameters to six. It has been shown [7] that a divergence of fitting results obtained by using general parametrization scheme and the superposition model is often negligible or of the same order as uncertainties of the 
experimental data. The most important off-superposition contribution originating from the dynamic coupling mechanism (dynamic ligand polarization) $[2,8,9]$ can be, in the case of hypersensitive transitions [8], estimated relatively easily from the first principles.

This paper deals with a simplified parametrization of the intensities in which the global interconfigurational matrix elements of an odd parity perturbation are expressed in terms of local ones associated with a single ligand-metal system. We adopt here the idea of angular overlap model (AOM) proposed many years ago by Schäffer and Jørgensen [10] for interpretation of even part of the crystal field potential and observed crystal field splittings. An essential role of the overlap and covalency mechanisms observed for the $f$-electron systems is reflected by an apparent regularity of the common AOM parameters [11]. One can expect these mechanisms to be even more important in the case of intensity parameters. It is promising that the two kinds of the interconfiguration excitations giving rise to the electric dipole transition, $f \rightarrow d$ and $f \rightarrow g$, are described by separate parameters. Clear physical sense of the usual AOM parameters has opened further possibilities of simplification of the model [11]. An adoption of the model to the static part of the intensity matrix is believed to give similar effects.

\section{Formulation of the model}

General expansion of the effective crystal field potential $V\left(r_{i}\right)$ acting on an electron $r_{i}$ of metal ion in terms of the spherical harmonics $C_{p}^{(t)}\left(r_{i} / r_{i}\right)$ reads

$$
V\left(r_{i}\right)=\sum_{t p} \beta_{t p}\left(r_{i}\right) C_{p}^{(t)}\left(r_{i} / r_{i}\right),
$$

where $\beta_{t p}(r)$ 's are the radial multipliers of the expansion. Replacing of the usual point charge model expansion with (1) results in the following modification of the well-known Judd intensity formulas [12]:

$$
A_{t p}\left\langle n l\left|r^{t}\right| n^{\prime} l^{\prime}\right\rangle \rightarrow\left\langle n l\left|\beta_{t p}(r)\right| n^{\prime} l^{\prime}\right\rangle \text {. }
$$

In turn, following the basic angular overlap model idea [11] we accept the superposition approximation according to which $V(r)$ is a superposition of independent individual ligand potentials $v_{L}\left(r_{L}\right), r_{L}=r-\boldsymbol{R}_{L}$, of $C_{\infty v}$ symmetry along each metal-ligand axis. Matrix elements of these local potentials in coordinate systems the $z$ axes of which are directed towards the $C_{\infty v}$ axes play a role of AOM parameters $e_{\mu}$ :

$$
e_{\mu}\left(n l, n^{\prime} l^{\prime} ; R_{L}\right)=\left\langle n l \mu\left|v_{L}\left(r_{L}\right)\right| n^{\prime} l^{\prime} \mu\right\rangle,
$$

where $|n l \mu\rangle$ and $\left|n^{\prime} l^{\prime} \mu\right\rangle$ denotes one-electron orbitals of the metal ion. In the conventional AOM parametrization both the $|n l \mu\rangle$ and $\left|n^{\prime} l^{\prime} \mu\right\rangle$ orbitals correspond to the open shell states $n f$ for the $f$-electron systems we are interested in. In the case of intensities only the matrix elements between the $n f$ states and those of opposite parity, $n d$ and $n g$, are of interest.

The global matrix elements of $V\left(r_{i}\right)$ are simply the sum of the ligand contributions (2) transformed to the common, global coordinate system

$$
\left\langle n l m|V(r)| n^{\prime} l^{\prime} m^{\prime}\right\rangle=\sum_{L \mu} D_{\mu m}^{(l)^{*}}(L) D_{\mu m^{\prime}}^{\left(l^{\prime}\right)}(L) e_{\mu}\left(n l, n^{\prime} l^{\prime} ; R_{L}\right),
$$


where $D_{\mu m}^{(l)}$ is the l-rank rotation matrix corresponding to ligand $L$. Combining (1) and (3) one obtains

$$
\begin{gathered}
\left\langle n l\left|\beta_{t p}(r)\right| n^{\prime} l^{\prime}\right\rangle=\frac{[t]}{\left\{[l]\left[l^{\prime}\right]\right\}^{1 / 2}}\left[\left(\begin{array}{ccc}
l & t & l^{\prime} \\
0 & 0 & 0
\end{array}\right)\right]^{-1} \sum_{L \mu}(-1)^{\mu}\left(\begin{array}{ccc}
l & t & l^{\prime} \\
-\mu & 0 & \mu
\end{array}\right) \\
\times C_{p}^{(t)^{*}}(L) e_{\mu}\left(n l, n^{\prime} l^{\prime} ; R_{L}\right)
\end{gathered}
$$

where the standard notation of $3 j$ symbols and the convention $[l] \equiv 2 l+1$ is introduced. $C_{p}^{(t)}(L) \equiv C_{p}^{(t)}\left(\Theta_{L}, \Phi_{L}\right)$, where $\Theta_{L}$ and $\Phi_{L}$ are the angular coordinates of ligand $L$ in the central coordinate system. Inserting of Eq. (4) into the standard Judd formulas gives the following expression for intensity parameters:

where

$$
\begin{aligned}
A_{t p}^{\lambda} & =[\lambda][t]^{1 / 2} \sum_{l^{\prime}}\left\{\begin{array}{lll}
1 & \lambda & t \\
l & l^{\prime} & l
\end{array}\right\} \sum_{\mu}(-1)^{\mu}\left(\begin{array}{ccc}
l & t & l^{\prime} \\
-\mu & 0 & \mu
\end{array}\right) \\
& \times \sum_{L} C_{p}^{(t)^{*}}(L) \varepsilon_{\mu}\left(l, l^{\prime}, R_{L}\right)
\end{aligned}
$$

$$
\varepsilon_{\mu}\left(l, l^{\prime}, R_{L}\right)=2\left\{[l]\left[l^{\prime}\right]\right\}^{1 / 2}\left(\begin{array}{lll}
l & 1 & l^{\prime} \\
0 & 0 & 0
\end{array}\right) \sum_{n^{\prime}} \frac{\left\langle n l|r| n^{\prime} l^{\prime}\right\rangle}{\Delta E_{n^{\prime} l^{\prime}}} e_{\mu}\left(n l, n^{\prime} l^{\prime}, R_{L}\right)
$$

are the effective AOM intensity parameters, $\lambda=2,4,6, t=\lambda \pm 1, \Delta E_{n^{\prime \prime} l^{\prime}}$ represents an energy gap between the configurations $l^{N}$ and $l^{N-1} l^{\prime}$. We follow here the Reid and Richardson notation [2] in which the effective vector operator $V$ representing the metal-ligand-radiation field interaction is expanded in terms of the vector unit tensors $\boldsymbol{U}_{t p}^{\lambda}$ :

$$
\boldsymbol{V}=\sum_{\lambda t p} A_{t p}^{\lambda} \boldsymbol{U}_{t p}^{\lambda}
$$

The $A_{t p}^{\lambda}$ 's relate to the original Judd parameters $A_{t p} \Xi(t, \lambda)$ as follows:

$$
A_{t p}^{\lambda}=-\frac{[\lambda]}{[t]^{1 / 2}} A_{t p} \Xi(t, \lambda) \text {. }
$$

There are three $\varepsilon$ parameters corresponding to the admixture of $n d$ states: $\varepsilon_{\sigma}(d), \varepsilon_{\pi}(d), \varepsilon_{\delta}(d)$ and four ones relating to $n g$ states: $\varepsilon_{\sigma}(g), \varepsilon_{\pi}(g), \varepsilon_{\delta}(g), \varepsilon_{\phi}(g)$. Due to the symmetry restrictions associated with the superposition approximation [2] only six of them are independent. By analogy with the usual AOM parametrization [11] we assume that they depend on the metal-ligand distance $R$ as $R^{-\alpha}, \alpha$ being in the range 6.0-10.0 (for the present calculations purpose we put $\alpha=8$ ), and that they are ordered according to their absolute values as listed above, i.e. $\varepsilon_{\sigma}(d)$ is the most important, $\varepsilon_{\pi}(d)$ - less and so on. The latter assumption is necessary to decide which of the parameters should be excluded as overnumerous in the case of limited experimental data.

\section{Examples and discussion}

In order to check the new parametrization scheme we reexamine several examples of lanthanide complexes considered previously by Reid et al. [4, 13], making use of the relation (5). The result of the least square fits of the $\varepsilon$ parameters to the experimental " $A_{t p}^{\lambda}$ 's is shown in Tables I-III. The calculations have been performed within the following two models: 
TABLE I

Intensity parameters obtained by least squares fitting of three $(A)$ and five (B) AOM parameters $\varepsilon^{a}$ to the experimental values for $\mathrm{Ho}^{3+}: \mathrm{YPO}_{4}\left(D_{2 d}\right)$ and $\operatorname{LiTmF}_{4}\left(S_{4}\right)$.

\begin{tabular}{c|c|c|c|c|c}
\hline \hline Para- & \multicolumn{2}{|c|}{$\mathrm{Ho}^{3+}: \mathrm{YPO}_{4}^{b}$} & \multicolumn{3}{c}{$\mathrm{LiTmF}_{4}^{b}$} \\
\cline { 2 - 6 } meter & Model $A$ & Model $B^{c}$ & Model $A$ & Model $B$ & Experiment \\
\hline$A_{32}^{2}$ & 40 & -66 & $0.20 \pm 0.03 \mathrm{i}$ & $1+0.16 \mathrm{i}$ & $1+0.16 \mathrm{i}$ \\
$A_{32}^{4}$ & 139 & 169 & $0.69+0.11 \mathrm{i}$ & $0.46+0.07 \mathrm{i}$ & $0.46+0.07 \mathrm{i}$ \\
$A_{52}^{4}$ & -234 & -234 & $0.91-0.33 \mathrm{i}$ & $0.91-0.33 \mathrm{i}$ & $1.16+0.36 \mathrm{i}$ \\
$A_{52}^{6}$ & 470 & 470 & 0 & 0 & 0 \\
$A_{72}^{6}$ & - & -115 & - & $-0.03-0.02 \mathrm{i}$ & $0.3+0.24 \mathrm{i}$ \\
$A_{76}^{6}$ & - & $(1586)$ & - & $-0.33+0.23 \mathrm{i}$ & $-0.55+0.06 \mathrm{i}$ \\
\hline$\varepsilon_{\sigma}(d)$ & 1 & 1 & 1 & 1 & \\
$\varepsilon_{\pi}(d)$ & 1.15 & 0.86 & 0.9 & 0.94 & \\
$\varepsilon_{\delta}(d)$ & 1.14 & 0.56 & 0.61 & 0.75 & \\
$\varepsilon_{\sigma}(g)$ & - & 0.1 & - & 0.12 & \\
$\varepsilon_{\pi}(g)$ & - & 0.23 & - & 0.09 & \\
\hline
\end{tabular}

${ }^{a}$ Relative magnitudes of $\varepsilon$ parameters are given.

${ }^{b}$ Experimental values of intensity parameters and coordination geometry data used in calculations taken from Ref. [4] and references therein $(i=\sqrt{-1})$.

${ }^{c}$ Fitting is exact, i.e. experimental values of the $A_{t p}^{\lambda}$ parameters are the same except $A_{76}^{6}$ (given in parentheses) which has been mistakenly considered in Ref. [4] as $A_{74}^{6}$ and therefore it has not been taken into account in the fitting.

TABLE II

Intensity parameters obtained by least squares fitting of three $(A)$ and five $(B)$ AOM parameters $\varepsilon^{a}$ to the experimental values for $\mathrm{Eu}\left(\mathrm{C}_{2} \mathrm{H}_{5} \mathrm{SO}_{4}\right)_{3} \cdot 9 \mathrm{H}_{2} \mathrm{O}$ and $\mathrm{Tm}\left(\mathrm{C}_{2} \mathrm{H}_{5} \mathrm{SO}_{4}\right)_{3} \cdot 9 \mathrm{H}_{2} \mathrm{O}$.

\begin{tabular}{c|c|c|c|c}
\hline \hline \multirow{2}{*}{$\begin{array}{c}\text { Para- } \\
\text { meter }\end{array}$} & \multicolumn{2}{|c|}{$\mathrm{Eu}\left(\mathrm{C}_{2} \mathrm{H}_{5} \mathrm{SO}_{4}\right)_{3} \cdot 9 \mathrm{H}_{2} \mathrm{O}^{b}$} & \multicolumn{2}{c}{$\mathrm{Tm}\left(\mathrm{C}_{2} \mathrm{H}_{5} \mathrm{SO}_{4}\right)_{3} \cdot 9 \mathrm{H}_{2} \mathrm{O}^{c}$} \\
\cline { 2 - 5 } & Model $A$ & Model $B^{d}$ & Model $A$ & Model $B^{d}$ \\
\hline$A_{33}^{2}$ & -0.08 & -1 & -0.11 & -43.7 \\
$A_{33}^{4}$ & -0.29 & -0.02 & -0.39 & 12 \\
$A_{53}^{4}$ & -4.32 & -4.32 & -14.5 & -14.5 \\
$A_{53}^{6}$ & 1.23 & 1.23 & 9.64 & 9.64 \\
$A_{73}^{6}$ & - & 1.26 & - & -7.55 \\
\hline$\varepsilon_{\sigma}(d)$ & 1 & 1 & 1 & 1 \\
$\varepsilon_{\pi}(d)$ & 0.97 & 0.94 & 0.97 & 0.83 \\
$\varepsilon_{\delta}(d)$ & 0.81 & 0.73 & 0.77 & 0.72 \\
$\varepsilon_{\sigma}(g)$ & - & 0.08 & - & 0.1 \\
$\varepsilon_{\pi}(g)$ & - & 0.05 & - & 0.06 \\
\hline
\end{tabular}

${ }^{a}$ Relative magnitudes of $\varepsilon$ are given.

$b, c$ Experimental values of intensity parameters and coordination geometry data taken from Ref. [13] and [4], respectively. Approximate $D_{3 h}$ symmetry has been assumed.

${ }^{d}$ Fitting is exact.

$A$ - three-parameter version of the AOM in which the parameters related to the $f^{N-1} n g$ configuration are excluded,

$B$ - five-parameter model allowing for $\varepsilon_{\sigma}(g)$ and $\varepsilon_{\pi}(g)$ apart from $\varepsilon_{\mu}(d)$ parameters. 
TABLE III

Intensity parameters obtained by least squares fitting of three $(A)$ and five $(B)$ AOM parameters $\varepsilon^{a}$ to the experimental values for $\mathrm{Pr}^{3+}: \mathrm{LaAlO}_{3}^{b}\left(D_{3}\right)$.

\begin{tabular}{c|c|c|c}
\hline \hline Parameter & Model $A$ & Model $B$ & Experiment \\
\hline$A_{33}^{2}$ & 4.18 & -2.8 & -2.8 \\
$A_{33}^{4}$ & 14.72 & 16.7 & 16.7 \\
$A_{53}^{4}$ & -17.1 & -17.1 & -17.1 \\
$A_{53}^{6}$ & 41.2 & 41.2 & 41.2 \\
$A_{73}^{6}$ & - & 4.92 & -2.3 \\
$A_{76}^{6}$ & - & -9.27 & -13.1 \\
\hline$\varepsilon_{\sigma}(d)$ & 1 & 1 & \\
$\varepsilon_{\pi}(d)$ & 1.02 & 1.00 & \\
$\varepsilon_{\delta}(d)$ & 0.8 & 0.8 & \\
$\varepsilon_{\sigma}(g)$ & - & 0.04 & \\
$\varepsilon_{\pi}(g)$ & - & -0.09 & \\
\hline
\end{tabular}

${ }^{a}$ Relative magnitudes of $\varepsilon$ parameters are given.

${ }^{b}$ Experimental values of intensity parameters and coordination geometry data taken from Ref. [4] and references therein. The phase $i$ common for all the $A_{t p}^{\lambda}$ parameters is immaterial.

As seen from the tables, the model $A$ well reproduces the $A_{t p}^{\lambda}$ parameters for $\lambda=4.6$ and $t=3.5 \mathrm{but}$, unexpectedly, for $\lambda=2$ it does not. Inclusion of $\varepsilon_{\mu}(g)$ parameters (model $B$ ) removes these discrepancies. Since the configuration interaction is only one of the possible intensity mechanisms (though strongly believed to be the essential one in most cases) there is not enough evidence for any physical significance of this observation. On the other hand, the apparent consistency of the $\varepsilon_{\mu}(d)$ parameters in the models $A$ and $B$ justifies the observed special role of the $\varepsilon_{\mu}(g)$ parameters.

Fixed ratios of the AOM parameters, as in the case of the common AOM applications [11], turn out to be a characteristic feature of the present results (see Table IV). It concerns mainly the $\varepsilon_{\mu}(d)$ parameters although a regularity of the

TABLE IV

AOM intensity parameters obtained for some RE compounds.

\begin{tabular}{|c|c|c|c|c|c|}
\hline & $\varepsilon_{\sigma}(d)$ & $\varepsilon_{\pi}(d)$ & $\varepsilon_{\delta}(d)$ & $\varepsilon_{\sigma}(g)$ & $\varepsilon_{\pi}(g)$ \\
\hline $\mathrm{Ho}^{3+}: \mathrm{YPO}_{4}$ & 1 & 0.86 & 0.56 & 0.1 & 0.23 \\
\hline $\operatorname{LiTmF}_{4}$ & 1 & 0.94 & 0.75 & 0.12 & 0.09 \\
\hline $\mathrm{Eu}\left(\mathrm{C}_{2} \mathrm{H}_{3} \mathrm{SO}_{4}\right)_{3} \cdot 9 \mathrm{H}_{2} \mathrm{O}$ & 1 & 0.94 & 0.73 & 0.08 & 0.05 \\
\hline $\operatorname{Tm}\left(\mathrm{C}_{2} \mathrm{H}_{3} \mathrm{SO}_{4}\right)_{3} \cdot 9 \mathrm{H}_{2} \mathrm{O}$ & 1 & 0.93 & 0.72 & 0.1 & 0.06 \\
\hline $\mathrm{Pr}^{3+}: \mathrm{LaAlO}_{3}$ & 1 & 1 & 0.8 & 0.04 & -0.09 \\
\hline average & 1 & 0.93 & 0.71 & 0.09 & 0.07 \\
\hline
\end{tabular}

$\varepsilon_{\mu}(g)$ parameters is also visible. Moreover, the latter parameters are of an order of magnitude lower than the corresponding $\varepsilon_{\mu}(d)$ 's. Comparing the obtained $\varepsilon_{\mu}(g)$ with their mean values from Table IV one can see that the largest divergences take place for $\mathrm{Pr}^{3+}: \mathrm{LaAlO}_{3}$ and $\mathrm{Ho}^{3+}: \mathrm{YPO}_{4}$ where the experimental $A_{7 p}^{6} / A_{7 p^{\prime}}^{6}$ ratios are clearly inconsistent with those required by the model. 
The observed proportions of the $\varepsilon$ parameters may have an universal character resulting from a specific spatial distribution of the particular $d, f$ and $g$ orbitals involved in the metal-ligand interaction. To verify this presumption much more examples should be considered making use of the experimental transition matrix elements determined directly from the intensity data rather than the phenomenological $A_{t p}^{\lambda}$ parameters. Further theoretical work should also be undertaken.

\section{Acknowledgments}

The impetus for this work was provided by Dr. J.C. Krupa, who is thanked for his kind interest and stimulating discussions.

\section{References}

[1] D.J. Newman, G. Ballasubramanian, J. Phys. C 8, 37 (1975).

[2] M.F. Reid, F.S. Richardson, J. Chem. Phys. 79, 5735 (1983).

[3] M. Kibler, J.C. Gâcon, Croat. Chem. Acta 62, 783 (1989).

[4] M.F. Reid, J.J. Dallara, F.S. Richardson, J. Chem. Phys. 79, 5743 (1983).

[5] M.F. Reid, Betty Ng, D.J. Newman, J. Less-Common Met. 148, 219 (1989).

[6] L. Smentek-Mielczarek, Phys. Rev. B 40, 6499 (1989).

[7] D.K.T. Chan, M.F. Reid, J. Less-Common Met. 148, 207 (1989).

[8] R.D. Peacock, Struct. Bonding 22, 83 (1975).

[9] B.R. Judd, Phys. Rev. 70, 4830 (1979).

[10] C.E. Schäffer, C.K. Jörgensen, Mol. Phys. 9, 401 (1965).

[11] Z. Gajek, J. Mulak, J. Phys., Condens. Matter 4, 427 (1992).

[12] B.R. Judd, Phys. Rev. 127, 750 (1962).

[13] M.F. Reid, F.S. Richardson, J. Chem. Phys. 80, 3507 (1984). 